\title{
COMPLEX STRUCTURES ON AFFINE MOTION GROUPS
}

\author{
MARÍA L. BARBERIS AND ISABEL G. DOTTI
}

\begin{abstract}
We study existence of complex structures on semidirect products $\mathfrak{g} \oplus_{\rho} \mathfrak{v}$ where $\mathfrak{g}$ is a real Lie algebra and $\rho$ is a representation of $\mathfrak{g}$ on $\mathfrak{v}$. Our first examples, the Euclidean algebra $\mathfrak{e}(3)$ and the Poincaré algebra $\mathfrak{e}(2,1)$, carry complex structures obtained by deformation of a regular complex structure on $\mathfrak{s l}(2, \mathbb{C})$. We also exhibit a complex structure on the Galilean algebra $\mathcal{G}(3,1)$. We construct next a complex structure on $\mathfrak{g} \oplus_{\rho} \mathfrak{v}$ starting with one on $\mathfrak{g}$ under certain compatibility assumptions on $\rho$.

As an application of our results we obtain that there exists $k \in\{0,1\}$ such that $\left(S^{1}\right)^{k} \times$ $E(n)$ admits a left invariant complex structure, where $S^{1}$ is the circle and $E(n)$ denotes the Euclidean group. We also prove that the Poincaré group $P^{4 k+3}$ has a natural left invariant complex structure.

In case $\operatorname{dim} \mathfrak{g}=\operatorname{dim} \mathfrak{v}$, then there is an adapted complex structure on $\mathfrak{g} \oplus_{\rho} \mathfrak{v}$ precisely when $\rho$ determines a flat, torsion-free connection on $\mathfrak{g}$. If $\rho$ is self-dual, $\mathfrak{g} \oplus_{\rho} \mathfrak{v}$ carries a natural symplectic structure as well. If, moreover, $\rho$ comes from a metric connection then $\mathfrak{g} \oplus_{\rho} \mathfrak{v}$ possesses a pseudo-Kähler structure.

We prove that the tangent bundle $T G$ of a Lie group $G$ carrying a flat torsion free connection $\nabla$ and a parallel complex structure possesses a hypercomplex structure. More generally, by an iterative procedure, we can obtain Lie groups carrying a family of left invariant complex structures which generate any prescribed real Clifford algebra.
\end{abstract}

\section{InTRODUCTION}

A complex structure on a real Lie algebra $\mathfrak{g}$ is an endomorphism $J$ of $\mathfrak{g}$ satisfying: $J^{2}=-1$ and $N_{J} \equiv 0$, where $N_{J}$ denotes the Nijenhuis tensor corresponding to $J$ (equation (2) below). This integrability condition is equivalent to a splitting of $\mathfrak{g}^{\mathbb{C}}=\mathfrak{g} \otimes_{\mathbb{R}} \mathbb{C}$ as

$$
\mathfrak{g}^{\mathbb{C}}=\mathfrak{g}^{1,0} \oplus \mathfrak{g}^{0,1}
$$

where $\mathfrak{g}^{1,0}$ (resp. $\mathfrak{g}^{0,1}$ ), the $i$-eigenspace (resp. - $i$-eigenspace) of $J$, is a complex Lie subalgebra. Observe that in case $\mathfrak{g}$ admits such a structure then its real dimension is even and $J$ induces a complex structure on any Lie group $G$ with Lie algebra $\mathfrak{g}$ such that left translations by elements of $G$ are holomorphic maps on $G$.

There are two particular cases when the integrability condition is satisfied:

- $\mathfrak{g}^{1,0}$ and $\mathfrak{g}^{0,1}$ are ideals of $\mathfrak{g}^{\mathbb{C}}$, which is equivalent to ad $(x) \circ J=J \circ$ ad $(x)$ for all $x \in \mathfrak{g}$, that is, $(\mathfrak{g}, J)$ is a complex Lie algebra;

- $\mathfrak{g}^{1,0}$ and $\mathfrak{g}^{0,1}$ are abelian subalgebras, which is equivalent to ad: $(\mathfrak{g}, J) \rightarrow\left(\mathfrak{g l}(\mathfrak{g}), R_{J}\right)$ being anti-holomorphic, and we say that the complex structure $J$ is abelian (cf. 3], [6]). Here, $R_{J}(u)=u \circ J, u \in \mathfrak{g l}(\mathfrak{g})$.

1991 Mathematics Subject Classification. Primary ; Secondary .

Key words and phrases.

Both authors were partially supported by CONICET, ANPCyT, SECYT-UNC and ACC (Argentina). 
Samelson showed in 23] that any even dimensional compact Lie algebra $\mathfrak{g}$ admits a complex structure, which is determined by considering a root space decomposition of $\mathfrak{g}^{\mathbb{C}}$. The case of reductive $\mathfrak{g}$ was considered by D. Snow in 24, where he classified the regular left invariant complex structures on $G$. For the solvable case there are partial results depending on $\operatorname{dim}_{\mathbb{R}} \mathfrak{g}$. If $\mathfrak{g}$ is solvable and $\operatorname{dim}_{\mathbb{R}} \mathfrak{g}=4$ the classification is complete: J. Snow classified in [25] all four dimensinal Lie algebras $\mathfrak{g}$ such that $\operatorname{dim}[\mathfrak{g}, \mathfrak{g}] \leq 2$ carrying a complex structure and $G$. Ovando extended in [21] this classification to the case $\operatorname{dim}[\mathfrak{g}, \mathfrak{g}]=3$. If $\operatorname{dim}_{\mathbb{R}} \mathfrak{g}=6$ and $\mathfrak{g}$ is nilpotent, S. Salamon determined all such Lie algebras admitting a complex structure and estimated the dimension of the moduli space of complex structures on $\mathfrak{g}$ (cf. [22).

In the present article we focus on the problem of finding complex structures on certain semidirect products $\mathfrak{g} \oplus_{\rho} \mathfrak{v}$ where $\rho: \mathfrak{g} \rightarrow \mathfrak{g l}(\mathfrak{v})$ is a Lie algebra homomorphism and we look upon $\mathfrak{v}$ as an abelian Lie algebra.

In Section 3 we obtain new results concerning natural complex structures on automorphism groups of differential geometric structures. For instance, we exhibit left invariant complex structures on the Euclidean group $E(3)$, the Poincaré group $P^{3}$ and the Galilean group $G(3,1)$ and we obtain new results concerning subgroups of $\operatorname{Aff}\left(\mathbb{R}^{n}\right)=\mathrm{GL}\left(\mathbb{R}^{n}\right) \ltimes \mathbb{R}^{n}$. Our main theorem in this section states that there exists $k \in\{0,1\}$ such that $\left(S^{1}\right)^{k} \times E(n)$ admits a complex structure. We point out that $\left(S^{1}\right)^{k} \times E(n)$ is not a complex Lie group. We prove an analogous result for the Poincaré group $P^{4 k+3}$.

In Section 4 we restrict ourselves to representations arising from an affine structure on $\mathfrak{g}$. Our interest in this situation is related to the fact that the structures we obtain provide a wide variety of examples which deserve a better understanding due to their relation with symplectic and special complex geometries. In fact, starting with a Lie algebra equipped with an affine structure we obtain a natural complex structure on its tangent algebra, which in addition is a special complex structure. Moreover, if the affine structure on $\mathfrak{g}$ defines a self-dual representation of $\mathfrak{g}$, the corresponding tangent algebra is special symplectic. A particular case occurs in the presence of a metric connection, thus obtaining a pseudo-Kähler structure. On the other hand, if $\mathfrak{g}$ possesses a complex structure $J$ and an affine structure $\nabla$ such that $\nabla J=0$, then the tangent algebra of $(\mathfrak{g}, \nabla)$ admits a hypercomplex structure. This procedure can be iterated to obtain Lie algebras possessing a family of complex structures generating a Clifford algebra of arbitrary order.

\section{Preliminaries}

We start by recalling the basic definitions. A complex structure on a real Lie algebra $\mathfrak{g}$ is an endomorphism $J$ of $\mathfrak{g}$ satisfying

$$
J^{2}=-1, \quad N_{J}(x, y)=0, \quad \forall x, y \in \mathfrak{g},
$$

where $N_{J}$ is the Nijenhuis tensor associated to $J$ :

$$
N_{J}(x, y)=J[x, y]-[J x, y]-[x, J y]-J[J x, J y],
$$

in other words, $J$ is integrable. By a hypercomplex structure we mean a pair of anticommuting complex structures. More generally, a $\mathrm{Cl}_{m}$-structure on $\mathfrak{g}$ is a family $\left\{J_{1}, \ldots, J_{m}\right\}$ of pairwise anticommuting complex structures on $\mathfrak{g}$ such that the real associative subalgebra of End $(\mathfrak{g})$ generated by $\left\{J_{1}, \ldots, J_{m}\right\}$ is isomorphic to the real Clifford algebra associated to $\mathbb{R}^{m}$ 
with the Euclidean inner product. Observe that a $\mathrm{Cl}_{2}$-structure is precisely a hypercomplex structure on $\mathfrak{g}$. $\mathrm{Cl}_{m}$-structures on certain solvable Lie algebras were constructed in 3 . If $G$ is a Lie group with Lie algebra $\mathfrak{g}$ then a $\mathrm{Cl}_{m}$-structure on $\mathfrak{g}$ can be left translated to all of $G$.

The following lemma, whose proof is straightforward, will be used in the sequel when proving that $N_{J} \equiv 0$.

Lemma 2.1. Let $\mathfrak{g}=\mathfrak{u} \oplus J \mathfrak{u}$ be a decomposition of $\mathfrak{g}$ where $\mathfrak{u}$ is a vector subspace. Then $N_{J}(u, v)=0, \quad \forall u, v \in \mathfrak{u}$ if and only if $N_{J} \equiv 0$.

We recall that an affine structure (or a left symmetric algebra structure) on a Lie algebra $\mathfrak{g}$ is a linear map $\rho: \mathfrak{g} \rightarrow \mathfrak{g l}(\mathfrak{g})$ satisfying the following conditions:

$$
\begin{aligned}
\rho[x, y] & =[\rho(x), \rho(y)], \\
\rho(x) y-\rho(y) x & =[x, y] .
\end{aligned}
$$

These conditions are satisfied if and only if

$$
\phi: \mathfrak{g} \rightarrow \mathfrak{a f f}(\mathfrak{g}), \quad x \mapsto \phi(x)=(\rho(x), x)
$$

is a Lie algebra homomorphism, where $\mathfrak{a f f}(\mathfrak{g})=\mathfrak{g l}(\mathfrak{g}) \oplus \mathfrak{g}$ is the Lie algebra of affine motions of $\mathfrak{g}$ when we look upon $\mathfrak{g}$ as a vector space. If $G$ is a Lie group with Lie algebra $\mathfrak{g}$, then affine structures on $\mathfrak{g}$ are in one to one correspondence with left invariant flat, torsion-free affine connections on $G$ (see [9]).

A symplectic structure on $\mathfrak{g}$ is a non-degenerate skew-symmetric bilinear form $\omega$ satisfying $\mathrm{d} \omega=0$, where

for $x, y, z \in \mathfrak{g}$.

$$
\mathrm{d} \omega(x, y, z)=\omega(x,[y, z])+\omega(y,[z, x])+\omega(z,[x, y])
$$

\section{Complex structures on AfFine motion Lie Algebras}

Let $\mathfrak{g}$ be a Lie algebra and $\mathfrak{v}$ a $\mathfrak{g}$-module, that is, there exists a Lie algebra homomorphism $\rho: \mathfrak{g} \rightarrow \mathfrak{g l}(\mathfrak{v})$. Let $\mathfrak{g} \oplus_{\rho} \mathfrak{v}$ denote the semidirect product of $\mathfrak{g}$ by $\mathfrak{v}$, where we look upon $\mathfrak{v}$ as an abelian Lie algebra. The bracket on $\mathfrak{g} \oplus_{\rho} \mathfrak{v}$ is given as follows:

$$
[(x, u),(y, v)]=([x, y], \rho(x) v-\rho(y) u) \quad \text { for } x, y \in \mathfrak{g}, u, v \in \mathfrak{v} .
$$

We will be studying complex structures on Lie algebras of the above type. The more general situation $\mathfrak{g} \oplus_{\rho} \mathfrak{r}$ where $\mathfrak{r}$ is a Lie algebra, $\mathfrak{D}(\mathfrak{r})$ is the space of derivations of $\mathfrak{r}$ and $\rho: \mathfrak{g} \rightarrow \mathfrak{D}(\mathfrak{r})$ is a Lie algebra homomorphism will be the object of future study.

In case $\rho$ is a representation of $\mathfrak{g}$ on itself we will denote by $\mathfrak{g}_{a}$ the vector space underlying $\mathfrak{g}$, and we refer to the semidirect product

$$
T_{\rho} \mathfrak{g}:=\mathfrak{g} \oplus_{\rho} \mathfrak{g}_{a}
$$

as the tangent algebra of $(\mathfrak{g}, \rho)$ (compare with Definition 2.9 in [10]).

Observe that if $G \subset \mathrm{GL}\left(\mathbb{R}^{n}\right)$ is a Lie subgroup with Lie algebra $\mathfrak{g}$, then setting $\rho=$ standard representation on $\mathbb{R}^{n}$, it follows that the Lie algebra structure on $\mathfrak{g} \oplus_{\rho} \mathbb{R}^{n}$ is that inherited from the Lie algebra $\mathfrak{a f f}\left(\mathbb{R}^{n}\right)=\mathfrak{g l}(n, \mathbb{R}) \oplus \mathbb{R}^{n}$ of affine motions, in other words, $\mathfrak{g} \oplus_{\rho} \mathbb{R}^{n}$ is a Lie subalgebra of $\mathfrak{a f f}\left(\mathbb{R}^{n}\right)$.

The next two subsections contain some motivating examples. 
3.1. Euclidean, Poincaré and Galilean algebras. Let $\mathfrak{s o}(3)$ act on $\mathfrak{v}=\mathbb{R}^{3}$ by the standard representation $\rho$ via left matrix multiplication on vector columns. Then $\mathfrak{s o}(3) \oplus_{\rho} \mathbb{R}^{3} \cong$ $\mathfrak{e}(3)$, the Euclidean algebra, admits a complex structure. In fact, let $h=e_{12}-e_{21}, f_{13}=$ $e_{13}-e_{31}, f_{23}=e_{23}-e_{32}$ be a basis of $\mathfrak{s o}(3)$, where $e_{i j}$ is the $3 \times 3$ matrix with 1 at the $(i, j)$ entry and 0 elsewhere. Let $e_{i}, 1 \leq i \leq 3$, be the canonical basis of $\mathbb{R}^{3}$. Define $J$ on $\mathfrak{e}(3)$ as follows:

$$
J h=e_{3}, \quad J f_{13}=f_{23}, \quad J e_{1}=e_{2} .
$$

The integrability of $J$ can be checked by direct calculation of $N_{J}$. This construction can be extended to $\mathfrak{e}(4 k+3)$ (Theorem 3.6 below).

Consider the Lie algebra $\mathfrak{s o}(2,1)$ of the Lorentz group acting on Minkowski spacetime $\mathbb{R}^{2,1}$ by the standard representation $\rho$. Then $\mathfrak{e}(2,1)=\mathfrak{s o}(2,1) \oplus_{\rho} \mathbb{R}^{2,1}$ is the Lie algebra of the Poincaré group $P^{3}$. Let $s_{i j}=e_{i j}+e_{j i}, i \neq j$ and define a complex structure $J$ on $\mathfrak{e}(2,1)$ as follows:

$$
J h=e_{3}, \quad J s_{13}=s_{23}, \quad J e_{1}=e_{2} .
$$

In this case the integrability of $J$ follows again by direct calculation of $N_{J}$. This can also be extended to $\mathfrak{e}(4 k+2,1)$ (Theorem 3.7 below).

It is well known that $\mathfrak{s o}(3,1) \cong \mathfrak{s l}(2, \mathbb{C})$ deforms into $\mathfrak{e}(3)$ and $\mathfrak{e}(2,1)$. Moreover, if $\mathfrak{s l}(2, \mathbb{C})$ has complex basis $\left\{H, X_{+}, X_{-}\right\}$with bracket relations

$$
\left[H, X_{+}\right]=2 X_{+},\left[H, X_{-}\right]=-2 X_{-}, \quad\left[X_{+}, X_{-}\right]=-H
$$

the complex structure given by

$$
J H=i H, \quad J X_{+}=i X_{+}, \quad J X_{-}=-i X_{-}
$$

is integrable and it is regular in the sense of 24]. Its associated complex subalgebra $\mathfrak{s o}(3,1)^{1,0}$ is solvable hence the above structure is not equivalent to the canonical one (see [27, where a classification of all homogeneous 6-dimensional homogeneous complex manifolds is given). Deforming

$$
\mathfrak{s l}(2, \mathbb{C})=\mathfrak{s o}(3)^{\mathbb{C}}=\mathfrak{s o}(2,1)^{\mathbb{C}}
$$

into $\mathfrak{e}(3)$ and $\mathfrak{e}(2,1)$, it is not hard to verify that under the above deformation of $\mathfrak{s l}(2, \mathbb{C})$ the complex structure $J$ considered above remains integrable along the deformation and yields the complex structures considered in $\mathfrak{e}(3)$ and $\mathfrak{e}(2,1)$.

Let $\mathcal{G}(3,1)$ be the Lie algebra of the Galilean group, that is,

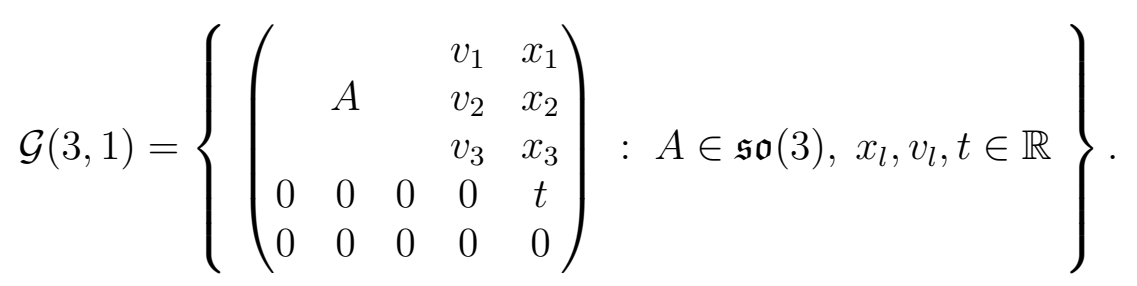

We show next that $\mathcal{G}(3,1)$ admits a complex structure. Let $\left\{h, f_{13}, f_{23}\right\}$ be the basis of $\mathfrak{s o}(3)$ as above and $\operatorname{span}\left\{e_{i}, e_{j}^{\prime}: 1 \leq i \leq 3,1 \leq j \leq 4\right\}$ a complementary subspace of $\mathfrak{s o}(3)$ 
in $\mathfrak{g}$. Note that setting $v_{l}=0, l=1,2,3$ and $t=0$ the resulting subalgebra is $\mathfrak{e}(3)$. Let $J$ be defined as follows:

$$
\begin{aligned}
J h & =e_{3}^{\prime}, & J e_{1}^{\prime}=e_{2}^{\prime}, & J e_{1}=e_{2}, \\
J f_{13} & =f_{23}, & J e_{3}=e_{4}^{\prime} . &
\end{aligned}
$$

The integrability of $J$ follows by checking directly the vanishing of $N_{J}$. The Galilean group can be thought of as the isometry group of $\mathbb{R}^{4}=\operatorname{span}\left\{e_{i}^{\prime}: 1 \leq i \leq 4\right\}$ with the degenerate metric $g=d x_{1}^{2}+d x_{2}^{2}+d x_{3}^{2}$, and it can be obtained as a deformation of the Poincaré algebra $\mathfrak{e}(3,1)$ (see [13).

The preceding paragraphs can be summarized as follows:

Proposition 3.1. The Euclidean, Poincaré and Galilean algebras $\mathfrak{e}(3), \mathfrak{e}(2,1)$ and $\mathcal{G}(3,1)$ admit complex structures such that

(1) the complex structures on $\mathfrak{e}(3)$ and $\mathfrak{e}(2,1)$ are deformations of a regular complex structure on $\mathfrak{s l}(2, \mathbb{C})$,

(2) the natural embedding $\mathfrak{e}(3) \hookrightarrow \mathcal{G}(3,1)$ is holomorphic.

We note that the above complex structure on $\mathfrak{e}(3)$ induces an invariant complex structure on $T S^{3}$, the tangent bundle of $S^{3}$. This follows from the isomorphism $\mathfrak{s o}(3) \oplus_{\rho} \mathbb{R}^{3} \cong \mathfrak{s o}(3) \oplus$ ad $\mathfrak{s o}(3)_{a}$ (see (6) ), since the standard representation $\rho$ (via left matrix multiplication) of $\mathfrak{s o}(3)$ on $\mathfrak{v}=\mathbb{R}^{3}$ is equivalent to the adjoint representation. We show next that this particular example can be generalized to $T G$ for an arbitrary Lie group $G$. On the other hand, in [18] a complex structure is given to the tangent bundle of any $n$-dimensional sphere by identifying $T S^{n}$ with the affine hyperquadric $V_{n}=\left\{\left(z_{1}, \ldots, z_{n+1}\right) \in C^{n+1}: \sum_{i} z_{i}^{2}=1\right\}$.

3.2. Tangent and cotangent bundle of a Lie group $G$ with an invariant complex structure. Both, the tangent and cotangent bundle of a Lie group $G$ are Lie groups in a natural way. In fact, identifying the tangent bundle $T G$ with $G \times \mathfrak{g}, T G$ has a natural Lie group structure as the semidirect product under the adjoint representation and the corresponding Lie algebra is the tangent algebra of $(\mathfrak{g}, \mathrm{ad})$ (see (6) ). Assume that $\mathfrak{g}$ has a complex structure $J$ and define $J_{+}$on $T_{\text {ad }} \mathfrak{g}=\mathfrak{g} \oplus_{\text {ad }} \mathfrak{g}_{a}$ by $J(x, v)=(J x, J v)$. Then $J_{+}$defines a complex structure on $T_{\text {ad }} \mathfrak{g}$. This easily follows by observing that $N_{J_{+}}((x, 0),(0, v))=$ $\left(0, N_{J}(x, v)\right)$, which vanishes by the integrability of $J$.

There is an analogous statement for the cotangent bundle $T^{*} G$ with the coadjoint representation. In a similar way, we get that $J_{+}$defines a complex structure on $\mathfrak{g} \oplus_{\mathrm{ad}^{*}} \mathfrak{g}^{*}$, where $J$ on $\mathfrak{g}^{*}$ is defined by $J(\alpha)(x)=-\alpha(J x)$ for $\alpha \in \mathfrak{g}^{*}, \quad x \in \mathfrak{g}$. The integrability of $J_{+}$on $T^{*} G$ is again a consequence of the integrability of $J$.

We summarize the preceding paragraphs as follows:

Proposition 3.2. Let $G$ be a Lie group equipped with an invariant complex structure J. Then the tangent and cotangent bundles, $T G$ and $T^{*} G$, carry natural globally defined invariant complex structures.

Remark 3.1. We note that for a complex manifold $(M, J)$, any given torsion free connection $\nabla$ on $M$ satisfying $\nabla J=0$ allows to construct $J_{+}$on $T M$ using the decomposition of $T(T M)=H \oplus V$, where $H$ is the horizontal distribution corresponding to the connection $\nabla$ 
and $V$ is the vertical distribution tangent to the fibers. On the other hand, $T^{*}(M)$ always carries a natural complex structure induced from that of $M$.

Remark 3.2. We show below (Proposition 3.3) that if ad is replaced by a representation $\rho$ of $\mathfrak{g}$ on itself satisfying certain compatibility conditions, $J_{+}$as above is integrable on $T G=G \times \mathfrak{g}$ with respect to the group structure determined by $\rho$. By considering the dual $\rho^{*}$ of $\rho$ we obtain the analogous result for the cotangent bundle $T^{*} G=G \times \mathfrak{g}^{*}$. The latter situation was considered in [12] for $\rho$ arising from the Obata connection associated to a hypercomplex structure on $G$.

subsectionMain results Let $\mathfrak{v}$ be a real vector space, $\operatorname{dim} \mathfrak{v}=2 n$, and fix a real endomorphism $I$ of $\mathfrak{v}$ satisfying $I^{2}=-$ id. Given a complex structure $J$ on $\mathfrak{g}$, consider on $\mathfrak{g} \oplus_{\rho} \mathfrak{v}$ two endomorphisms $J_{+}$and $J_{-}$defined by

$$
J_{ \pm}(x, v)=(J x, \pm I v) \quad \text { for } x \in \mathfrak{g}, v \in \mathfrak{v} .
$$

We study next the integrability of $J_{ \pm}$on $\mathfrak{g} \oplus_{\rho} \mathfrak{v}$. This result will be used throughout this section.

Proposition 3.3. Let $(\mathfrak{g}, J)$ be a Lie algebra with a complex structure $J$ admitting a decomposition $\mathfrak{g}=\mathfrak{g}_{0} \oplus \mathfrak{g}_{1}$ with $\mathfrak{g}_{k}$ J-stable subspaces, $k=0,1$. Assume that $\mathfrak{g}$ acts on a complex vector space $(\mathfrak{v}, I)$ and the action $\rho: \mathfrak{g} \rightarrow \mathfrak{g l}(\mathfrak{v})$ satisfies the following compatibility conditions:

(i) $\rho(x) J=J \rho(x)$ for all $x \in \mathfrak{g}_{0}$;

(ii) $\rho(J x) I v=\rho(x) v$ for all $x \in \mathfrak{g}_{1}, v \in \mathfrak{v}$.

Then, $J_{+}$is integrable on $\mathfrak{g} \oplus_{\rho} \mathfrak{v}$. If $\mathfrak{g}_{1}=0$, then $J_{-}$is also integrable.

Proof. The following statements are easily proved by a direct calculation of $N_{J_{+}}$and $N_{J_{-}}$, respectively:

$$
\begin{aligned}
& N_{J_{+}}((x, 0),(0, v))=0 \quad \text { if and only if } \quad[I, \rho(x)] v=[I, \rho(J x)] I v, \\
& N_{J_{-}}((x, 0),(0, v))=0 \quad \text { if and only if }[I, \rho(x)] v=-[I, \rho(J x)] I v,
\end{aligned}
$$

for $x \in \mathfrak{g}, \quad v \in \mathfrak{v}$. The proposition now follows from these observations.

Combining the above proposition with Samelson's result (cf. 23]) we obtain:

Corollary 3.4. Let $\mathfrak{g}$ be a compact Lie algebra acting on $\mathbb{C}^{n}$ by complex linear maps. Then there exists $s \in\{0,1\}$ such that $\mathbb{R}^{s} \oplus\left(\mathfrak{g} \oplus_{\rho} \mathbb{C}^{n}\right)$ admits a complex structure.

Remark 3.3. The above corollary has a corresponding analogue in the hypercomplex case, replacing $\mathbb{C}^{n}$ by $\mathbb{H}^{n}$ with $\mathfrak{g}$ acting by quaternionic linear maps and allowing $s \in\{0,1,2,3\}$ in Corollary [3.4. In fact, Joyce proved in [15] that given a compact Lie algebra $\mathfrak{g}$ there exists $s \in\{0,1,2,3\}$ such that $\mathbb{R}^{s} \oplus \mathfrak{g}$ admits a hypercomplex structure. Combining this fact with Proposition 3.3 we obtain the desired generalization to the hypercomplex case.

The following result is a particular case of Proposition 3.3 where $\mathfrak{g}=\mathfrak{g}_{1}$.

Corollary 3.5. $\mathfrak{a f f}\left(\mathbb{R}^{2 n}\right)$ admits a complex structure. 
Proof. Fix a real endomorphism $I$ of $\mathbb{R}^{2 n}$ satisfying $I^{2}=-\mathrm{id}$ and let us denote by $R_{I}$ the endomorphism of $\mathfrak{g l}\left(\mathbb{R}^{2 n}\right)$ defined by

$$
R_{I}(u)=u \circ I, \quad u \in \mathfrak{g l}\left(\mathbb{R}^{2 n}\right) .
$$

It is straightforward that $R_{I}$ defines a complex structure on $\mathfrak{g l}\left(\mathbb{R}^{2 n}\right)$, that is, $R_{I}$ is integrable (see also [16], §4, Example 1). In fact, identifying $\mathfrak{g l}\left(\mathbb{R}^{2 n}\right) \cong \mathbb{R}^{4 n^{2}} \cong \mathbb{C}^{2 n^{2}}$, it turns out that $R_{I}$ is the complex structure induced by multiplication by $i$ on $\mathbb{C}^{2 n^{2}}$. Let $J=R_{-I}$ and define $J_{+}$on $\mathfrak{a f f}\left(\mathbb{R}^{2 n}\right)=\mathfrak{g l}\left(\mathbb{R}^{2 n}\right) \oplus \mathbb{R}^{2 n}$ as in (17). The corollary now follows from Proposition 3.3 applied to $\mathfrak{g}=\mathfrak{g l}\left(\mathbb{R}^{2 n}\right), \mathfrak{v}=\mathbb{R}^{2 n}$ and $\rho=$ standard representation, by observing that $\mathfrak{g}_{0}=0$.

We prove below the main result in this section, namely, that the isometry group of the Euclidean $n$-space, $E(n)$, carries invariant complex structures when it is even dimensional $(n \equiv 0,3(4))$; when the dimension is odd $(n \equiv 1,2(4)) S^{1} \times E(n)$ carries invariant complex structures. For $n \equiv 0,1$ (4) the proof follows by applying Proposition 3.3 with a given complex structure on $\mathfrak{s o}(n)$. For $n \equiv 2(4)$, Proposition 3.3 applies again starting with a complex structure on $\mathbb{R} z \oplus \mathfrak{s o}(n)$. The case $n \equiv 3$ (4) differs from the others since $\mathfrak{s o}(n)$ is odd dimensional so it cannot be holomorphically embedded in $\mathfrak{e}(n)$. We get around this difficulty by fixing a toral subalgebra $\mathfrak{t}$ in $\mathfrak{s o}(n)$, an element $h \in \mathfrak{t}$ and define $J$ so that $J h$ belongs to the centralizer of $h$ in $\mathfrak{e}(n)$.

The Lie algebra of $E(n)$ is the Euclidean algebra $\mathfrak{e}(n) \cong \mathfrak{s o}(n) \oplus_{\rho} \mathbb{R}^{n}$, where $\rho$ is the standard representation of $\mathfrak{s o}(n)$ on $\mathbb{R}^{n}$ by left matrix multiplication on vector columns. Before stating the theorem we introduce a complex structure $J$ on $\mathfrak{s o}(n), n \equiv 0,1(4)$, that will be needed later. Let $e_{i j}$ denote the square matrix with entry 1 at the $(i, j)$ entry, all other entries being 0 . Then

$$
\left[e_{i j}, e_{r s}\right]=\delta_{j r} e_{i s}-\delta_{s i} e_{r j} \text {. }
$$

Set $f_{i j}=e_{i j}-e_{j i}, h_{i}=f_{2 i-1,2 i}$ and let $n=4 k$ or $4 k+1$, so that $\mathfrak{s o}(n)$ has rank $2 k$. Define $J$ on $\mathfrak{s o}(n)$ such that $J^{2}=-1$ as follows:

$$
J h_{i}=h_{i+1}, 1 \leq i \leq 2 k, \quad J f_{2 j-1, l}=f_{2 j, l}, 2 \leq 2 j<l \leq n .
$$

The integrability of $J$ easily follows from the root space decomposition of $\mathfrak{s o}(n, \mathbb{C}$ ) (see [14]). Observe that the trivial central extension $\mathbb{R} z \oplus \mathfrak{s o}(4 k+2)$ admits a complex structure which is obtained by (12) and $J h_{2 k+1}=z$.

Theorem 3.6. Let $\mathfrak{e}(n)$ be the Euclidean Lie algebra. Then there exists $s \in\{0,1\}$ such that $\mathbb{R}^{s} \oplus \mathfrak{e}(n)$ has a complex structure and the following inclusions are holomorphic for any nonnegative integer $k$ :

$$
\mathfrak{e}(4 k) \hookrightarrow \mathfrak{e}(4 k+1) \oplus \mathbb{R} e_{4 k+2} \hookrightarrow \mathfrak{e}(4 k+2) \oplus \mathbb{R} e_{4 k+3} \hookrightarrow \mathfrak{e}(4 k+3) .
$$

Proof. Let $n=4 k$, where $k \geq 1$ is an integer and let $e_{l}$ be the vector column whose only non-zero entry is 1 in the $l$ th coordinate, $1 \leq l \leq 4 k$. Let $I$ be the complex structure on $\mathbb{R}^{4 k}$ satisfying $I e_{2 i-1}=e_{2 i}, \quad 1 \leq i \leq 2 k$ and $J$ the complex strucure on $\mathfrak{s o}(n)$ defined in equation (12). We show next that $\mathfrak{g}=\mathfrak{s o}(4 k)$ splits as $\mathfrak{g}=\mathfrak{g}_{0} \oplus \mathfrak{g}_{1}$ where $\mathfrak{g}_{0}$ and $\mathfrak{g}_{1}$ are $J$ invariant 
subspaces satisfying the hypothesis of Proposition 3.3 and therefore $J_{+}$defines a complex structure on $\mathfrak{e}(4 k)$. Set

$$
\begin{gathered}
h_{i}=f_{2 i-1,2 i}, \quad 1 \leq i \leq 2 k, \\
u_{j l}^{ \pm}=f_{2 j-1,2 l-1} \pm f_{2 j, 2 l}, \quad v_{j l}^{ \pm}=f_{2 j-1,2 l} \pm f_{2 j, 2 l-1}, \quad 1 \leq j<l \leq 2 k,
\end{gathered}
$$

and let

$$
\begin{gathered}
\mathfrak{g}_{0}=\operatorname{span}_{\mathbb{R}}\left\{h_{i}: 1 \leq i \leq 2 k\right\} \oplus \operatorname{span}_{\mathbb{R}}\left\{u_{j l}^{+}, v_{j l}^{-}: 1 \leq j<l \leq 2 k\right\}, \\
\mathfrak{g}_{1}=\operatorname{span}_{\mathbb{R}}\left\{u_{j l}^{-}, v_{j l}^{+}: 1 \leq j<l \leq 2 k\right\} .
\end{gathered}
$$

Observe that $\mathfrak{g}_{0}$ is a subalgebra isomorphic to $\mathfrak{u}(2 k)$ and therefore condition (i) of the proposition is satisfied. It remains to show that $\left(J u_{j l}^{-}\right)(I w)=u_{j l}^{-} w$ for all $w=\sum_{i=1}^{4 k} w_{i} e_{i}$ in $\mathbb{R}^{4 k}$, $1 \leq j<l \leq 2 k$. We calculate

$$
\begin{aligned}
\left(J u_{j l}^{-}\right)(I w) & =v_{j l}^{+}\left(\sum_{i=1}^{2 k}\left(-w_{2 i} e_{2 i-1}+w_{2 i-1} e_{2 i}\right)\right) \\
& =w_{2 l-1} e_{2 j-1}-w_{2 l} e_{2 j}-w_{2 j-1} e_{2 l-1}+w_{2 j} e_{2 l}=u_{j l}^{-} w
\end{aligned}
$$

which implies that condition (ii) of the proposition is satisfied, therefore $J_{+}(x, w)=(J x, I w)$ is a complex structure on $\mathfrak{e}(4 k)$.

We show next that the trivial central extension $\mathbb{R} z \oplus \mathfrak{e}(4 k+1)$ admits a complex structure. Let $\mathfrak{p}=\operatorname{span}_{\mathbb{R}}\left\{f_{i, 4 k+1}: 1 \leq i \leq 4 k\right\}$, so that

$$
\mathfrak{e}(4 k+1)=\mathfrak{e}(4 k) \oplus \mathfrak{p} \oplus \mathbb{R} e_{4 k+1} .
$$

We define $J$ on $\mathbb{R} z \oplus \mathfrak{e}(4 k+1)$ so that its restriction to $\mathfrak{e}(4 k)$ is the complex structure defined above, $J$ restricted to $\mathfrak{s o}(4 k+1)$ is given by equation (12) and

$$
J e_{4 k+1}=z .
$$

We show next that $J$ is integrable. It is straightforward that $N_{J}\left(x, e_{4 k+1}\right)=0$ for $x \in \mathfrak{e}(4 k)$. In view of Lemma 2.1. we only need to check that $N_{J}\left(f_{2 i-1,4 k+1}, e_{2 j-1}\right)=0,1 \leq i \leq k, 1 \leq$ $j \leq k+1$. It will be convenient to write down the following brackets, which will be used to carry out the calculations

$$
\left[f_{i, 4 k+1}, e_{4 k+1}\right]=e_{i}, \quad\left[f_{i, 4 k+1}, f_{j, 4 k+1}\right]=f_{j i}, \quad i \neq j \quad\left[f_{i, 4 k+1}, e_{j}\right]=-\delta_{i j} e_{4 k+1},
$$

for $1 \leq i, j \leq 4 k$.

$$
\begin{aligned}
N_{J}\left(f_{2 i-1,4 k+1}, e_{2 j-1}\right)= & J\left[f_{2 i-1,4 k+1}, e_{2 j-1}\right]-\left[J f_{2 i-1,4 k+1}, e_{2 j-1}\right]-\left[f_{2 i-1,4 k+1}, J e_{2 j-1}\right] \\
& -J\left[J f_{2 i-1,4 k+1}, J e_{2 j-1}\right] \\
= & -\delta_{i j} J e_{4 k+1}-\left[f_{2 i, 4 k+1}, e_{2 j-1}\right]-\left[f_{2 i-1,4 k+1}, e_{2 j}\right]-J\left[f_{2 i, 4 k+1}, e_{2 j}\right] \\
= & -\delta_{i j} J e_{4 k+1}+\delta_{i j} J e_{4 k+1}=0, \quad 1 \leq i, j \leq 2 k, \\
N_{J}\left(f_{2 i-1,4 k+1}, e_{4 k+1}\right)= & J\left[f_{2 i-1,4 k+1}, e_{4 k+1}\right]-\left[J f_{2 i-1,4 k+1}, e_{4 k+1}\right]-\left[f_{2 i-1,4 k+1}, J e_{4 k+1}\right] \\
& -J\left[J f_{2 i-1,4 k+1}, J e_{4 k+1}\right] \\
= & J e_{2 i-1}-\left[f_{2 i, 4 k+1}, e_{4 k+1}\right]-\left[f_{2 i-1,4 k+1}, z\right]-J\left[f_{2 i, 4 k+1}, z\right] \\
= & J e_{2 i-1}-e_{2 i}=0, \quad 1 \leq i \leq 2 k,
\end{aligned}
$$


therefore, $J$ defines a complex structure on $\mathbb{R} z \oplus \mathfrak{e}(4 k+1)$.

The case of $\mathfrak{e}(4 k+2)$ is analogous to $\mathfrak{e}(4 k)$. The only difference is that $\mathfrak{s o}(4 k+2)$ has odd rank equal to $2 k+1$, so in this case we get that the trivial central extension $\mathbb{R} z \oplus \mathfrak{e}(4 k+2)$ has a complex structure. The complex structure on $\mathfrak{g}=\mathfrak{s o}(4 k+2) \oplus \mathbb{R} z$ is that defined after (12)). Let $\mathfrak{g}=\mathfrak{s o}(4 k+2) \oplus \mathbb{R} z$ where $z$ is central and let $h_{i}, 1 \leq i \leq 2 k+1, u_{j l}^{ \pm}, v_{j l}^{ \pm}, 1 \leq j<l \leq$ $2 k+1$ be defined as in (13) and (14). We define $I$ on $\mathbb{R}^{4 k+2}$ by $I e_{2 i-1}=e_{2 i}, \quad 1 \leq i \leq 2 k+1$. Proposition 3.3 applies again with

$$
\begin{gathered}
\mathfrak{g}_{0}=\operatorname{span}_{\mathbb{R}}\left\{h_{i}: 1 \leq i \leq 2 k+1\right\} \oplus \operatorname{span}_{\mathbb{R}}\left\{u_{j l}^{+}, v_{j l}^{-}: 1 \leq j<l \leq 2 k+1\right\} \oplus \mathbb{R} z \\
\mathfrak{g}_{1}=\operatorname{span}_{\mathbb{R}}\left\{u_{j l}^{-}, v_{j l}^{+}: 1 \leq j<l \leq 2 k+1\right\}
\end{gathered}
$$

and we obtain that $J_{+}$is a complex structure on $\mathbb{R} z \oplus \mathfrak{e}(4 k+2)$.

The case of $\mathfrak{e}(4 k+3)$ is similar to $\mathfrak{e}(4 k+1)$. Setting $\mathfrak{e}(4 k+3)=\mathfrak{e}(4 k+2) \oplus \mathbb{R} e_{4 k+3} \oplus \mathfrak{p}$, where $\mathfrak{p}=\operatorname{span}_{\mathbb{R}}\left\{f_{i, 4 k+3}: 1 \leq i \leq 4 k+2\right\}$ it turns out that the endomorphism $J$ on $\mathfrak{e}(4 k+3)$ restricting to the complex structure on $\mathfrak{e}(4 k+2) \oplus \mathbb{R} e_{4 k+3}$ defined above and satisfying $J f_{2 i-1,4 k+3}=f_{2 i, 4 k+3}, 1 \leq i \leq 2 k+1$. The integrability of $J$ follows by analogous arguments to those in the case of $\mathfrak{e}(4 k+1) \oplus \mathbb{R} z$.

Remark 3.4. (i) Observe that if $(M, g)$ is a compact riemannian manifold, then the isometry group $I(M, g)$ is a compact Lie group, hence it always admits a complex structure if it is even dimensional (cf. 23]).

(ii) If $M$ is a compact complex manifold then the group of holomorphic diffeomorphisms of $M, \mathfrak{H}(M)$, is a complex Lie group (cf. [7]). Moreover, if $M$ is Kähler-Einstein with non-zero Ricci tensor, then the Lie algebra of infinitesimal isometries is a real form of the Lie algebra $\mathfrak{h}(M)$ of the complex Lie group $\mathfrak{H}(M)$ (cf. [17).

The fact that the Galilean group admits a complex structure suggests that Theorem 3.6 can possibly be extended to groups of transformations preserving other geometric structures. Indeed, the following theorem shows that the isometry group of Minkowski space time $\mathbb{R}^{4 k+2,1}$ with the Lorentz metric possesses a natural complex structure. Before stating the result, we introduce a complex structure $J$ on $\mathfrak{s o}(4 k+2,1) \oplus \mathbb{R} e_{4 k+3}$ which will be needed in the proof of the theorem. The restriction of $J$ to $\mathfrak{s o}(4 k+2) \oplus \mathbb{R} e_{4 k+3}$ is the complex structure defined after (12), and its restriction to the subspace span $\left\{s_{i, 4 k+3}=e_{i, 4 k+3}+e_{4 k+3, i}, 1 \leq i \leq 4 k+2\right\}$ is defined as follows:

$$
J s_{2 i-1,4 k+3}=s_{2 i, 4 k+3}, \quad 1 \leq i \leq 2 k+1 .
$$

The integrability of $J$ follows by observing that both, $\mathfrak{s o}(4 k+3, \mathbb{C})^{1,0}$ and $\mathfrak{s o}(4 k+3, \mathbb{C})^{0,1}$, are complex subalgebras of $\mathfrak{s o}(4 k+3, \mathbb{C})$.

Theorem 3.7. The Lie algebra $\mathfrak{e}(4 k+2,1)$ of the Poincaré group $P^{4 k+3}$ admits a complex structure such that the embedding $\mathfrak{e}(4 k+2) \oplus \mathbb{R} e_{4 k+3} \hookrightarrow \mathfrak{e}(4 k+2,1)$ is holomorphic.

Proof. Let $J$ be the complex structure on $\mathfrak{s o}(4 k+2,1) \oplus \mathbb{R} e_{4 k+3}$ defined in the previous paragraph and let $I$ on $\mathbb{R}^{4 k+2}$ the canonical complex structure defined by $I e_{2 i-1}=e_{2 i}, 1 \leq$ $i \leq 2 k+1$. It follows that $J_{+}(x, v)=(J x, I v), x \in \mathfrak{s o}(4 k+2,1) \oplus \mathbb{R} e_{4 k+3}, v \in \mathbb{R}^{4 k+2}$ is 
integrable on $\mathfrak{e}(4 k+2,1)$. We apply Proposition 3.3 on $\mathfrak{g}=\mathfrak{s o}(4 k+2,1) \oplus \mathbb{R} e_{4 k+3}$ as follows:

$$
\begin{gathered}
\mathfrak{g}_{0}=\operatorname{span}_{\mathbb{R}}\left\{h_{i}: 1 \leq i \leq 2 k+1\right\} \oplus \operatorname{span}_{\mathbb{R}}\left\{u_{j l}^{+}, v_{j l}^{-}: 1 \leq j<l \leq 2 k+1\right\} \oplus \mathbb{R} e_{4 k+3}, \\
\mathfrak{g}_{1}=\operatorname{span}_{\mathbb{R}}\left\{u_{j l}^{-}, v_{j l}^{+}: 1 \leq j<l \leq 2 k+1\right\} \oplus \operatorname{span}_{\mathbb{R}}\left\{s_{i, 4 k+3}: 1 \leq i \leq 4 k+2\right\},
\end{gathered}
$$

where $h_{i}, u_{j l}^{ \pm}, v_{j l}^{ \pm}$were defined in (13) and (14).

The second assertion is a consequence of Theorem 3.6. since the restriction of $J_{+}$to $\mathfrak{e}(4 k+2) \oplus \mathbb{R} e_{4 k+3}$ is the complex structure we exhibited in the proof of that theorem.

\section{Complex And SympleCtiC STRUCTURES ON TANGEnT AlGEBras}

In this section we will restrict to the case $\mathfrak{v}=\mathfrak{g}_{a}$, where $\mathfrak{g}$ is the Lie algebra of a connected Lie group $G$, and $\rho$ a representation of $\mathfrak{g}$ on itself. Now, one may ask whether the endomorphism $K(x, y)=(y,-x)$ gives rise to a complex structure on $\mathfrak{g} \oplus_{\rho} \mathfrak{g}_{a}$. Related to this situation we considered in [6] the following family of Lie algebras.

Let $A$ be an associative algebra, let $\mathfrak{v}=A_{a}$ and $\rho: A \rightarrow \mathfrak{g l}(A)$, where $\rho(a)$ is left multiplication by $a$ in $A$. The bracket on $A \oplus_{\rho} A_{a}$ is given as follows:

$$
[(a, b),(c, d)]=(a c-c a, a d-c b), \quad a, b, c, d \in A .
$$

and a canonical complex structure $K$ is given by

$$
K(a, b)=(b,-a), \quad a, b \in A .
$$

This Lie algebra was denoted by $\mathfrak{a f f}(A)$ in [6].

Note that in the above family, the linear map $\rho$ satisfies equations (3) and (4), in other words, $\rho$ defines an affine structure on the induced Lie algebra $A$ (see Section 2). In this section such representations will be denoted by $\nabla$ due to their relation with flat connections on Lie groups.

A flat connection on a Lie algebra $\mathfrak{g}$ is a linear map $\nabla: \mathfrak{g} \rightarrow \mathfrak{g l}(\mathfrak{g}), \quad x \mapsto \nabla_{x}$ satisfying (3), in other words, $\nabla$ is a representation. If, moreover, (4) is satisfied we say that $\nabla$ is torsion-free and therefore the bracket in $\mathfrak{g}$ comes from the left symmetric algebra structure determined by $\nabla$.

We consider next tangent algebras $T_{\nabla} \mathfrak{g}$ for some real Lie algebra $\mathfrak{g}$ and some flat connection $\nabla: \mathfrak{g} \rightarrow \mathfrak{g l}(\mathfrak{g})$ (equation (6) $)$.

We show below that the endomorphism $K$ given by (21) defines a complex structure on $T_{\nabla} \mathfrak{g}$ precisely when the flat connection $\nabla$ is torsion-free (compare with results in [11]).

Theorem 4.1. Let $\mathfrak{g}$ be a real Lie algebra and $\nabla$ a flat affine connection on $\mathfrak{g}$. Then $K(x, y)=(y,-x)$ defines a complex structure on $T_{\nabla} \mathfrak{g}$ if and only if $\nabla$ is torsion-free. Moreover, if $\nabla$ is torsion-free then the connection $\nabla^{1}$ on $T_{\nabla} \mathfrak{g}$ defined by

$$
\nabla_{(x, y)}^{1}(z, w)=\left(\nabla_{x} z, \nabla_{x} w\right)
$$

is a flat, torsion-free connection such that $\nabla^{1} K=0$.

Conversely, if $\mathfrak{u}$ is a Lie algebra with a complex structure $K$ such that $\mathfrak{u}$ decomposes as $\mathfrak{u}=\mathfrak{g} \oplus K \mathfrak{g}$ where $\mathfrak{g}$ is a subalgebra and $K \mathfrak{g}$ is an ideal of $\mathfrak{u}$, then $K \mathfrak{g}$ is abelian and there exists a flat, torsion-free connection $\nabla$ on $\mathfrak{g}$ such that $\mathfrak{u}$ is the tangent algebra of $(\mathfrak{g}, \nabla)$. 
Proof. Integrability of $K$ gives $\left(\nabla_{x} y, 0\right)=K[(x, 0)(0, y)]=\left([x, y]+\nabla_{y} x, 0\right)$, that is, $\nabla$ is torsion-free. On the other hand, if $\nabla$ is torsion-free one computes:

$$
[K(x, 0),(y, 0)]+\left[((x, 0), K(y, 0)]=\left(0, \nabla_{y} x-\nabla_{x} y\right)\right.
$$

and

$$
K([(x, 0),(y, 0)]-[K(x, 0), K(y, 0)])=(0,-[x, y]),
$$

hence, $K$ is integrable on $T_{\nabla} \mathfrak{g}$. The assertion on $\nabla^{1}$ follows by direct calculation, using the properties of $\nabla$.

To prove the converse, since $\mathfrak{u}=\mathfrak{g} \oplus K \mathfrak{g}$ with $\mathfrak{g}$ a subalgebra and $K \mathfrak{g}$ an ideal, the map $x \mapsto-K \circ \operatorname{ad}(x) \circ K$ from $\mathfrak{g}: \rightarrow \mathfrak{g l}(\mathfrak{g})$ is a flat connection on $\mathfrak{g}$. Moreover, the integrability of $K$ toghether with the fact that $K \mathfrak{g}$ is an ideal gives $K \mathfrak{g}$ is an abelian ideal. Finally, if $x, y \in \mathfrak{g}$ one has

$$
-K \circ \operatorname{ad}(x) \circ K y+K \circ \operatorname{ad}(y) \circ K x=[x, y]
$$

since $K$ is integrable and $K \mathfrak{g}$ is abelian. Thus $\nabla_{x}=-K \circ \operatorname{ad}(x) \circ K$ defines an affine structure on $\mathfrak{g}$ and $\mathfrak{u}=T_{\nabla} \mathfrak{g}$, as claimed.

Remark 4.1. The above proposition is related with results in 2] concerning complex product structures on Lie algebras. We recall that a complex product structure on $\mathfrak{u}$ is a pair $J, E$ where $J$ is a complex structure, $E$ is an endomorphism of $\mathfrak{u}$ anticommuting with $J$ such that $E^{2}=\mathrm{id}$ and $\mathfrak{u}$ splits as $\mathfrak{u}=\mathfrak{u}_{+} \oplus \mathfrak{u}_{-}$where $\mathfrak{u}_{ \pm}$, the eigenspaces of $E$ of eigenvalue \pm 1 , are subalgebras of $\mathfrak{u}$. It turns out that $\mathfrak{u}_{-}=J \mathfrak{u}_{+}$. In [2] it is shown that when one of the eigenspaces of $E$, say $\mathfrak{u}_{-}$, is an ideal of $\mathfrak{u}$, then $\mathfrak{u}_{-}$is abelian so that, in view of the above proposition, $\mathfrak{u}$ is the tangent algebra of $\left(\mathfrak{u}_{+}, \nabla\right)$ where $\nabla$, the adjoint representation of $\mathfrak{u}$ restricted to $\mathfrak{u}_{+}$, is a flat torsion free connection on $\mathfrak{u}_{+}$. The above proposition says that the class of tangent algebras $T_{\nabla} \mathfrak{g}$ with $\nabla$ a flat torsion free connection on $\mathfrak{g}$ is in one to one correspondence with the class of Lie algebras $\mathfrak{u}$ admitting a complex product structure in which either $\mathfrak{u}_{+}$or $\mathfrak{u}_{-}$is an ideal of $\mathfrak{u}$.

Corollary 4.2. Let $G$ be a Lie group admitting a left invariant flat and torsion-free connection. Then, its tangent bundle $T G=G \times \mathfrak{g}$ carries a homogeneous complex structure which is parallel with respect to a flat torsion-free connection on TG and such that the embedding of $G$ in $G \times \mathfrak{g}$ is totally real.

As a special case of the above corollary we can quote the following various examples of Lie algebras carrying affine structures: $k$-step nilpotent Lie algebras, $k<4, \mathfrak{g l}\left(\mathbb{R}^{n}\right), \mathfrak{a f f}\left(\mathbb{R}^{n}\right)$, Lie algebras admitting non singular derivations.

Remark 4.2. The Lie groups with Lie algebra $T_{\nabla} \mathfrak{g}, \nabla$ a flat,torsion-free connection on $\mathfrak{g}$, are particular examples of special complex manifolds, that is, complex manifolds $(M, J)$ together with a flat torsion free connection $\nabla$ such that $\nabla J$ is symmetric (cf. 1).

Given a hypercomplex structure $\mathcal{H}=J_{1}, J_{2}$ on $\mathfrak{g}$ there exists a unique torsion-free connection $\nabla^{\mathcal{H}}$, called the Obata connection associated with $\mathcal{H}$, such that $\nabla^{\mathcal{H}} J_{1}=\nabla^{\mathcal{H}} J_{2}=0$ (see [20]). Furthermore, when $\nabla^{\mathcal{H}}$ is flat, it turns out that any Lie group with Lie algebra $\mathfrak{g}$ admits an atlas of charts such that coordinate changes are affine maps with quaternionic linear part (see [26]). The above theorem together with results in the previous section give: 
Corollary 4.3. Let $\mathfrak{g}$ be a real Lie algebra with a flat, torsion-free connection $\nabla$ and a parallel complex structure $J$. Then $T_{\nabla} \mathfrak{g}$ carries a hypercomplex structure whose Obata connection is $\nabla^{1}$ as in Theorem 4.1.

Proof. The hypercomplex structure is given by

$$
J_{-}(x, y)=(J x,-J y), \quad K(x, y)=(y,-x) .
$$

Observe that the condition $\nabla J=0$ is equivalent to condition (i) of Proposition 3.3 relative to $I=J$, which ensures the integrability of $J$. The integrability of $K$ follows from Theorem 4.1 . Also, $\nabla^{1} J_{=} \nabla^{1} K=0$, hence, by uniqueness, $\nabla^{1}$ coincides with the Obata connection, as claimed.

Combining previous results with the above corollary we obtain a hypercomplex structure on $T \operatorname{Aff}\left(\mathbb{C}^{n}\right)$, the tangent bundle of $\operatorname{Aff}\left(\mathbb{C}^{n}\right)$, and $T \mathrm{GL}\left(\mathbb{R}^{2 n}\right)$, the tangent bundle of $\mathrm{GL}\left(\mathbb{R}^{2 n}\right)$.

Corollary 4.4. TAff $\left(\mathbb{C}^{n}\right)$ and $T G L\left(\mathbb{R}^{2 n}\right)$ carry natural hypercomplex structures.

Proof. Consider the Lie algebra $\mathfrak{a f f}\left(\mathbb{C}^{n}\right)=\mathfrak{g l}\left(\mathbb{C}^{n}\right) \oplus \mathbb{C}^{n}$ of $\operatorname{Aff}\left(\mathbb{C}^{n}\right)$ with the flat torsion free connection $\nabla$ defined by $\nabla_{(x, v)}(y, u)=(x y, x u), x, y \in \mathfrak{g l}\left(\mathbb{C}^{n}\right), u, v \in \mathbb{C}^{n}$, and the complex structure $J_{+}$introduced in Corollary 3.5 which restricts to $\mathfrak{a f f}\left(\mathbb{C}^{n}\right)$. It can be checked that $\nabla J_{+}=0$ and the first assertion follows from Corollary 4.3 .

For the second case, let $J=R_{I}$ be the complex structure on $\mathfrak{g l}\left(\mathbb{R}^{2 n}\right)$ defined in (10) and $\nabla$ the standard flat torsion free connection on $\mathfrak{g l}\left(\mathbb{R}^{2 n}\right)$ defined by $\nabla_{(x, z)}(y, w)=(x y, x w)$, $x, y, z, w \in \mathfrak{g l}\left(\mathbb{R}^{2 n}\right)$. We can apply again Corollary 4.3 since $\nabla J=0$.

4.1. The cotangent algebra. Let $\nabla$ be a flat connection on $\mathfrak{g}$ and consider the contragredient representation $\nabla^{*}: \mathfrak{g} \rightarrow \mathfrak{g l}\left(\mathfrak{g}^{*}\right)$, that is:

$$
\nabla_{x}^{*} \alpha=-\alpha \circ \nabla_{x}, \quad x \in \mathfrak{g}, \alpha \in \mathfrak{g}^{*} .
$$

Consider the 2 -form $\Omega$ on the cotangent algebra $T_{\nabla}^{*} \mathfrak{g}:=\mathfrak{g} \oplus_{\nabla^{*}} \mathfrak{g}^{*}$ defined by

$$
\Omega((x, \alpha),(y, \beta))=\alpha(y)-\beta(x) .
$$

The following result is known (compare with Theorem 4.1 above); it shows that the conditions of $K$ being integrable on the tangent algebra and $\Omega$ being closed on the cotangent algebra are equivalent.

Proposition 4.5 ( 8] ). Let $\mathfrak{g}$ be a real Lie algebra, $\nabla$ a flat affine connection on $\mathfrak{g}$ and $\nabla^{*}$ the contragredient representation. Then $\Omega$ is closed on $T_{\nabla}^{*} \mathfrak{g}$ if and only if $\nabla$ is torsion-free.

We wish to study conditions which allow to induce structures from the cotangent algebra to the tangent algebra. For instance, let $\psi: \mathfrak{g} \rightarrow \mathfrak{g}^{*}$ be a vector space isomorphism and let $\omega$ be the 2 -form on $T_{\nabla} \mathfrak{g}$ induced by $\Omega$ and $\psi$, that is,

$$
\omega\left((x, y),\left(x^{\prime}, y^{\prime}\right)=\Omega\left((x, \psi y),\left(x^{\prime}, \psi y^{\prime}\right), \quad x, y, x^{\prime}, y^{\prime} \in \mathfrak{g} .\right.\right.
$$

It turns out that, in case $\psi$ is a $\mathfrak{g}$-module isomorphism between $(\mathfrak{g}, \nabla)$ and $\left(\mathfrak{g}, \nabla^{*}\right)$, then the non-degenerate 2 -form $\omega$ is closed, that is, it is a symplectic structure on $T_{\nabla} \mathfrak{g}$. In other 
words, the natural symplectic structure on the cotangent algebra can be transferred to the tangent algebra when the connection is self-dual.

Proposition 4.6. Let $\nabla$ be a flat torsion-free connection on $\mathfrak{g}$ and assume that $\psi:(\mathfrak{g}, \nabla) \rightarrow$ $\left(\mathfrak{g}, \nabla^{*}\right)$ is a $\mathfrak{g}$-module isomorphism. Then the 2 -form $\omega$ defined as in (22) is a symplectic structure on $T_{\nabla} \mathfrak{g}$ such that $\nabla^{1} \omega=0$.

Remark 4.3. Recall from [1] that a special symplectic structure on a manifold $M$ is a triple $(J, \nabla, \omega)$ where $\nabla$ is a flat torsion free connection, $J$ is a complex structure on $M$ such that $\nabla J$ is symmetric and $\omega$ is a parallel symplectic structure on $M$. In particular, $(J, \nabla)$ is a special complex structure on $M$. It follows from the above proposition and Theorem 4.1 that $\left(T_{\nabla} \mathfrak{g}, K, \nabla^{1}, \omega\right)$ is a particular case of a special symplectic structure.

A particular case of the above theorem occurs in the presence of a metric connection.

Let $\mathfrak{g}$ be a real Lie algebra equipped with a flat, torsion-free connection $\nabla$ and assume that $\nabla$ is a metric connection, that is, there is a non-degenerate symmetric bilinear form $\langle$,$\rangle on \mathfrak{g}$ having $\nabla$ as its Levi-Civita connection. In this case there is a natural isomorphism between $\mathfrak{g}$ and its dual $\mathfrak{g}^{*}$ :

$$
\psi: \mathfrak{g} \rightarrow \mathfrak{g}^{*}, \quad x \mapsto x^{b}
$$

where $x^{b}(y)=\langle x, y\rangle, \quad x, y \in \mathfrak{g}$. It follows that $\psi$ satisfies the hypothesis of Proposition 4.6 and therefore $T_{\nabla} \mathfrak{g}$ carries a natural symplectic structure $\omega$. Observe that the complex structure $K$ from Theorem 4.1 and the symplectic form $\omega$ on $T_{\nabla} \mathfrak{g}$ are related as follows:

$$
\left\langle K(x, y),\left(x^{\prime}, y^{\prime}\right)\right\rangle=\omega\left((x, y),\left(x^{\prime}, y^{\prime}\right)\right),
$$

that is, $(K, \omega)$ defines a pseudo-Kähler structure on $T_{\nabla} \mathfrak{g}$.

We summarize the above paragraph as follows:

Corollary 4.7. Let $\langle$,$\rangle be a flat metric on \mathfrak{g}$ and let $\nabla$ denote its Levi-Civita connection. Then $T_{\nabla} \mathfrak{g}$ possesses a natural pseudo-Kähler structure.

4.2. Clifford structures. Theorem 4.1 suggests that one could obtain $\mathrm{Cl}_{m}$-structures for arbitrary $m$ by an inductive procedure. In fact, this follows by observing that if we start with a Lie algebra $\mathfrak{g}$ equipped with a flat, torsion-free connection $\nabla$ then we can define $\nabla^{1}$ on $T_{\nabla} \mathfrak{g}$ as follows:

$$
\nabla_{(x, y)}^{1}(z, w)=\left(\nabla_{x} z, \nabla_{x} w\right)
$$

and $\nabla^{1}$ turns out to be a flat, torsion-free connection on the tangent algebra $T_{\nabla} \mathfrak{g}$ such that $\nabla^{1} K=0$. Set $T_{\nabla}^{1} \mathfrak{g}=T_{\nabla} \mathfrak{g}$ and for $m>1$ we define inductively $T_{\nabla}^{m} \mathfrak{g}$ to be the tangent algebra of $\left(T_{\nabla}^{m-1} \mathfrak{g}, \nabla^{m-1}\right)$, that is:

$$
T_{\nabla}^{m} \mathfrak{g}=T_{\nabla^{m-1}} T_{\nabla}^{m-1} \mathfrak{g}, \quad \nabla_{(u, v)}^{m}\left(u^{\prime}, v^{\prime}\right)=\left(\nabla_{u}^{m-1} u^{\prime}, \nabla_{u}^{m-1} v^{\prime}\right)
$$

Then $\nabla^{m}$ is a flat, torsion-free connection on $T_{\nabla}^{m} \mathfrak{g}$. The next theorem follows, by induction on $m$, from Theorem 4.1 and Corollary 4.3 by observing that Corollary 4.3 is the first step of the induction process. The second assertion follows from Corollary 4.7

Theorem 4.8. Let $\mathfrak{g}$ be a real Lie algebra carrying a flat, torsion-free connection $\nabla$. Then $\left(T_{\nabla}^{m} \mathfrak{g}, \nabla^{m}\right)$ carries a parallel $C l_{m}$-structure. Moreover, if $\nabla$ is a metric connection then 
there is a metric $g^{m}$ on $T_{\nabla}^{m}$ such that $\left(T_{\nabla}^{m}, g^{m}, J\right)$ is pseudo Kähler for all $J \in C l_{m}$ satisfying $J^{2}=-1$.

\section{REFERENCES}

(1) D.V. Alekseevsky, V. Cortés and C. Devchand, Special complex manifolds, J. Geom. Phys. 42 (1-2) (2002), 85-105; math.DG/9910091

(2) A. Andrada and S. Salamon, Complex product structures on Lie algebras, preprint 2003.

(3) M. L. Barberis, I. G. Dotti Miatello and R. J. Miatello, On certain locally homogeneous Clifford manifolds, Ann. Glob. Anal. Geom. 13 (1995), 289-301.

(4) M. L. Barberis, Abelian hypercomplex structures on central extensions of H-type Lie algebras, J. Pure Appl. Algebra 158 (2001), 15-23.

(5) M. L. Barberis and I. Dotti Miatello, Hypercomplex structures on a class of solvable Lie groups, Quart. J. Math. Oxford (2), 47 (1996), 389-404.

(6) M. L. Barberis and I. Dotti, Abelian complex structures on solvable Lie algebras, math.RA/0202220, to appear in J. Lie theory.

(7) S. Bochner and D. Montgomery, Groups on analytic manifolds, Ann. of Math. 48 (1947), 659-669.

(8) N. Boyom, Varietes symplectiques affines, Manuscripta Math. 64 (1989), 1-33.

(9) D. Burde, Affine structures on nilmanifolds, Int. J. Math. 7 (5), 1996, 599-616.

(10) J. M. Dardié and A. Médina, Algebres de Lie kählériennes et double extension, J. Algebra 185 (3) (1996), 774-995.

(11) P. Dombrowski, On the geometry of the tangent bundle, J. Reine Angew. Math. 210 (1962), $73-88$

(12) A. Fino, Cotangent bundle of hypercomplex 4-dimensional Lie groups, Manuscripta Math., to appear.

(13) Guillemin and S. Sternberg, Symplectic techniques in Physics, Cambridge University Press, 1984.

(14) S. Helgason, Differential geometry, Lie groups and symmetric spaces, Academic Press, 1978.

(15) D. Joyce, Compact hypercomplex and quaternionic manifolds, J. Differential Geometry 35 (1992), 743-761.

(16) D. Joyce, Manifolds with many complex structures, Quart. J. Math. Oxford (2), 46 (1995), 169-184.

(17) Y. Matsushima Sur la structure du groupe d'homéomorphismes analytiques d'une certaine variété kählérienne, Nagoya Math. J. 11 (1957), 145-150.

(18) A. Morimoto and T. Nagano, On pseudo-conformal transformation of hypersurfaces, J. Math. Soc. Japan, 15 (1963), 289-300.

(19) S. B. Myers and N. Steenrod, The group of isometries of a riemannian manifold, Ann. of Math. 40 (1939), 400-416.

(20) M. Obata, Affine connections on manifolds with almost complex, quaterion or Hermitian structures, Japan. J. Math. 26 (1956), 43-79.

(21) G. Ovando, Invariant complex structures on solvable real Lie groups, Manuscripta Math. 103 (2000), 19-30.

(22) S. Salamon, Complex structures on nilpotent Lie algebras, J. Pure Appl. Algebra 157 (2001), 311-333.

(23) H. Samelson, A class of complex analytic manifolds, Portugal. Math. 12 (1953), 129-132. 
(24) D. Snow, Invariant complex structures on reductive Lie groups, J. reine angew. Math. 371 (1986), 191-215.

(25) J. E. Snow, Invariant complex structures on four dimensional solvable real Lie groups, Manuscripta Math. 66 (1990), 397-412.

(26) A. Sommese, Quaternionic manifolds, Math. Ann. 212 (1975), 191-214.

(27) J. Winkelmann, The classification of three-dimensional homogeneous complex manifolds, Lecture Notes in Mathematics 1602, Springer Verlag (1995).

Ciem, Famaf, Universidad Nacional de Córdoba, Ciudad Universitaria, (5000) Córdoba, ARgENTINA

E-mail address: barberis@mate.uncor.edu

E-mail address: idotti@mate.uncor.edu 